KIAS 05036

\title{
Hadronic production and decays of Charginos and Neutralinos in Split Supersymmetry
}

\author{
Kingman Cheung \\ Department of Physics and NCTS, National \\ Tsing Hua University, Hsinchu, Taiwan, R.O.C. \\ Jeonghyeon Song \\ Department of Physics, Konkuk University, Seoul 143-701, Korea
}

(Dated: December 25, 2018)

\begin{abstract}
In the scenario recently proposed by Arkani-Hamed and Dimopoulos, the supersymmetric scalar particles are all very heavy, at least of the order of $10^{9} \mathrm{GeV}$ but the gauginos, higgsino, and one of the CP-even neutral Higgs bosons remain light under a TeV. In addition to gluino production, the first SUSY signature would be pair production of neutralinos and/or charginos. Here we study, with explicit $\mathrm{CP}$ violation, the production of neutralinos and charginos at hadronic colliders as well as the possible decay modes. We notice that the branching ratio of the neutralino radiative decay can be sizable unlike the case with light sfermions. In particular, the decay of $\widetilde{\chi}_{3}^{0} \rightarrow \widetilde{\chi}_{1}^{0} \gamma$ has a branching ratio of the order $O(1)$ percent. In colliders, it would give rise to signatures of an isolated single photon plus missing energy.
\end{abstract}

PACS numbers: 12.60.Jv, 14.80.Ly, 13.87.Fh 


\section{INTRODUCTION}

Supersymmetry (SUSY) is one of the most elegant solutions, if not the best, to the gauge hierarchy problem. It also provides a dynamical mechanism for electroweak symmetry breaking, as well as a viable candidate for dark matter (DM). Conventional wisdom tells us that SUSY must exist at the TeV scale, otherwise the fine tuning problem returns. Unfortunately these weak scale SUSY models, represented by the minimal supersymmetric standard model (MSSM), also have a number of difficulties such as SUSY flavor and CP problems, and the light Higgs boson. For the past two decades, particle theorists have made every effort to rectify the problems. Recently, Arkani-Hamed and Dimopoulos [1] adopted a rather radical approach to SUSY: they discarded the hierarchy problem and accepted the fine-tuning solution to the Higgs boson mass. They argued that much more serious finetuning is required for the observed small cosmological constant. If the cosmological constant problem is to be explained by the anthropic principle [2], an enormous number of metastable vacua, usually called the vast landscape of string theory vacua, are essential. Fine-tuning in the Higgs boson mass is more natural in this anthropic landscape [3].

Once we accept this proposal the finely-tuned Higgs boson mass is not a problem anymore. More concerned issue is to find a consistent set of parameters satisfying the following observations: (i) the refined result of the DM density to be $\Omega_{\mathrm{DM}} h^{2}=0.094-0.129$ (2 $\sigma$ range) by Wilkenson Microwave Anisotropy Probe (WMAP) [4]; (ii) sub-eV neutrino masses; and (iii) cosmological constant. The last one is accepted as an extremely fine-tuned principle. The second observation requires heavy right-handed neutrinos of a mass scale of $10^{11-13} \mathrm{GeV}$; it does not have appreciable effects on electroweak scale physics. The first observation, on the other hand, requires a weakly interacting particle of mass $\lesssim 1 \mathrm{TeV}$, in general. It is this requirement which affects most of the parameter space of the split SUSY scenario [5, 6].

The split SUSY scenario can be summarized as follows:

1. All scalars, except for a CP-even neutral Higgs boson, are super heavy, for which a common mass scale $\tilde{m}$ is assumed around $10^{9} \mathrm{GeV}$ to $M_{\mathrm{GUT}}$. Dangerous phenomena such as flavor-change neutral currents, CP-violating processes, and large electric dipole moments in the MSSM become safe. ${ }^{1}$

\footnotetext{
${ }^{1}$ There is another source of $C P$-violation as pointed out in Ref. 7].
} 
2. The gaugino and the Higgsino masses (the $\mu$ parameter), which can be much lighter than $\tilde{m}$ due to an $R$ and a PQ symmetry, respectively, are assumed near the TeV scale. Light supersymmetric fermions have additional virtue of gauge coupling unification as in MSSM, as well as providing a good DM candidate.

3. A light Higgs boson is very similar to the SM Higgs boson, but could be substantially heavier than that of the MSSM [1, [5, 8, 9, 10].

4. The DM density requires sufficient mixing in the neutralino sector, so that the lightest neutralino can efficiently annihilate [5, 11, 12]. In most cases, the $\mu$ parameter is relatively small.

Super heavy masses of SUSY scalar particles practically close off many neutralino annihilation channels, resulting in smaller annihilation cross section than that in MSSM. Giudice and Romanino [5], Pierce 11], and Masiero et al. 12] identified three interesting regions of the parameter space where the split SUSY model can accommodate the WMAP data on the DM density.

(i) The lightest neutralino is mostly a Bino, but with a substantial mixing with the Higgsino: $\mu$ is comparable to $M_{1}$. This is because the Bino interacts only with the Higgs and Higgsino when all sfermions are super heavy. Substantial mixing can guarantee efficient annihilation of the Bino into Higgs or $Z$ bosons. The WMAP data allow the gaugino mass parameters as low as the current lower bound.

(ii) The second region is where the LSP is mostly Higgsino with $M_{1,2} \gg \mu$. In this case, the Higgsino LSP annihilates via gaugino-Higgsino-Higgs and Higgsino-Higgsino-gauge couplings. Note that the lightest chargino and the second lightest neutralino are also predominately Higgsino since $\mu \ll M_{1,2}$, and thus degenerate in mass with the LSP. Efficient annihilation and co-annihilation require a rather heavy LSP mass $\mu \sim 1-1.2$ $\mathrm{TeV}$ for the LSP to be the DM.

(iii) The third region is the wino LSP in anomaly mediation and in this case $M_{2}<M_{1}, \mu$. Neutral wino can annihilate efficiently via an intermediate $W^{*}$, even in the absence of sfermions. Thus, a rather heavy wino with $M_{2} \simeq 2.0-2.5 \mathrm{TeV}$ is required to account for the dark matter density. 
The last two cases require a rather heavy LSP. Therefore, the chance of observing supersymmetric partners at colliders diminishes as the gluino would be even heavier than $2-3 \mathrm{TeV}$, though it is not impossible. Thus, we concentrate on the phenomenologically more interesting case where $M_{1} \sim \mu \sim M_{\mathrm{EW}}$. Note that once we impose the condition of gaugino-mass unification, $M_{2} \simeq 2 M_{1}$ at the weak scale. The current chargino mass bound is almost 104 $\mathrm{GeV}$ [13]. If converting to the bounds on $M_{2}$ and $\mu$, both $M_{2}$ and $\mu$ larger than $O(100) \mathrm{GeV}$ should be consistent with the bound. We do not specifically state the bound on $M_{1}$ and $\mu$, but $O(100) \mathrm{GeV}$ should be very safe for $M_{1}$ and $\mu$ with the chargino mass bound.

The detailed phenomenological study of the split SUSY scenario, especially focused on high energy colliders, is crucial to clarify the basic structure of the scenario. As pointed out in Ref. 1], the first unique feature is the stable or metastable gluino. It may give rise to stable charged tracks [14, 15, 16] or gluinonium [17] signatures. We focus on the neutralino pair, chargino pair, and neutralino-chargino pair production, and their decays at hadron colliders. These production channels may be the only accessible ones if the gluino mass is more than $3 \mathrm{TeV}$. There have been some studies of chargino and neutralino production at $e^{-} e^{+}$colliders [18] and at hadron colliders [14]. Some variations on split SUSY were also studied, e.g., taking $\mu$ to be very large [19], taking gaugino masses to be very large [20], or even taking $\mu$ and all gaugino masses to be very heavy [21]. ${ }^{2}$ Note that the phenomenology of split SUSY is rather similar to those of focus-point SUSY [22], except for the gluino phenomenology. There is also the PeV scale SUSY scenario [23].

At hadron colliders, the production of $\widetilde{\chi}_{i}^{0} \widetilde{\chi}_{j}^{0}, \widetilde{\chi}_{j}^{0} \widetilde{\chi}_{i}^{ \pm}$, and $\widetilde{\chi}_{i}^{+} \widetilde{\chi}_{j}^{-}$is mainly via the DrellYan-like processes with intermediate $W^{*}, \gamma^{*}$, or $Z^{*}$ exchanges as in the usual MSSM. The $t$-channel exchanges via $\tilde{q}, \tilde{\ell}$ are virtually absent. On the other hand, the decays of the neutralino $\tilde{\chi}_{j}^{0}(j>1)$ and chargino would be quite different from the MSSM case. With super-heavy sfermions the decay of the heavier neutralino $\tilde{\chi}_{j}^{0}$ into a lighter neutralino $\widetilde{\chi}_{i}^{0}$ plus $f \bar{f}$ is mainly via an intermediate $Z^{*}$ (the Higgsino component only) instead of a $\tilde{f}$. Another channel of importance is $\widetilde{\chi}_{j}^{0} \rightarrow \widetilde{\chi}_{i}^{ \pm} W^{*} \rightarrow \widetilde{\chi}_{i}^{ \pm} f \bar{f}^{\prime}$. We also have $\widetilde{\chi}_{j}^{0} \rightarrow \widetilde{\chi}_{i}^{0} h^{(*)} \rightarrow$ $\tilde{\chi}_{i}^{0}+\left(b \bar{b}\right.$ or $\left.W W^{*}\right)$. Finally, we note that the branching ratio of the radiative decay $\widetilde{\chi}_{j}^{0} \rightarrow \widetilde{\chi}_{i}^{0} \gamma$ can be sizable unlike the case with light sfermions. Phenomenologically it leaves clean signal

\footnotetext{
2 The last scenario was called supersplit supersymmetry which appeared in arXic.org on April fool's day. It is equivalent to the SM.
} 
at the LHC, consisting of a high-energy single-photon plus missing energy. Particularly in the parameter region where $|\mu|$ is comparable to $M_{1}$, the radiative branching ratio would be maximal. The chargino decays mainly through $\widetilde{\chi}_{j}^{+} \rightarrow \widetilde{\chi}_{i}^{0}+W^{*} \rightarrow \widetilde{\chi}_{i}^{0}+f \bar{f}^{\prime}$. By combining the phenomenological studies on neutralinos and charginos, as well as those of gluino, one may be able to distinguish the scenarios of split SUSY, PeV scale SUSY, or focus-point SUSY.

Improvement over previous studies [14, 18] can be summarized as follows.

1. We include all decay modes of neutralinos and charginos, in particular, the radiative decay mode. It gives rise to a single-photon plus missing energy signal. Experimentally, it is very clean.

2. We include the CP-violating phases in $M_{1}$ and $\mu$ in order to examine the effect of the $\mathrm{CP}$ phases on the decay of neutralinos.

3. We calculate the photon transverse momentum and missing transverse momentum distributions for the single-photon plus missing energy events, which mainly come from the decay of $\widetilde{\chi}_{3}^{0} \rightarrow \widetilde{\chi}_{1}^{0} \gamma$.

The organization of this paper is as follows. We describe the general formalism and convention in Sec. II. We calculate the decays and production cross sections for neutralino and chargino pairs in Sec. III and IV, respectively. We conclude in Sec. V.

\section{GENERAL FORMALISM OF NEUTRALINOS AND CHARGINOS}

The neutralino and chargino sectors are determined by fundamental SUSY parameters: the $\mathrm{U}(1)$ and $\mathrm{SU}(2)$ gaugino masses $M_{1}$ and $M_{2}$, the Higgsino mass parameter $\mu$, and $\tan \beta=v_{2} / v_{1}$ (the ratio between the vacuum expectation values of the two neutral Higgs fields). The neutralino mass matrix in the $\left(\widetilde{B}, \widetilde{W}^{3}, \widetilde{H}_{1}^{0}, \widetilde{H}_{2}^{0}\right)_{L}$ basis, where the subscript $L$ denotes the left-handed chirality of neutralinos, is [24]

$$
\mathcal{M}_{N}=\left(\begin{array}{cccc}
M_{1} & 0 & -m_{Z} c_{\beta} s_{W} & m_{Z} s_{\beta} s_{W} \\
0 & M_{2} & m_{Z} c_{\beta} c_{W} & -m_{Z} s_{\beta} c_{W} \\
-m_{Z} c_{\beta} s_{W} & m_{Z} c_{\beta} c_{W} & 0 & -\mu \\
m_{Z} s_{\beta} s_{W} & -m_{Z} s_{\beta} c_{W} & -\mu & 0
\end{array}\right)
$$


where $s_{\beta}=\sin \beta, c_{\beta}=\cos \beta$ and $s_{W}, c_{W}$ are the sine and cosine of the electroweak mixing angle $\theta_{W}$. Since $\mathcal{M}_{N}$ is symmetric, one unitary matrix $N$ can diagonalize the $\mathcal{M}_{N}$ such that $\mathcal{M}_{\text {diag }}=N^{*} \mathcal{M}_{N} N^{\dagger}$. The Majorana mass eigenstates are

$$
\left(\widetilde{\chi}_{1}^{0}, \widetilde{\chi}_{2}^{0}, \widetilde{\chi}_{3}^{0}, \widetilde{\chi}_{4}^{0}\right)_{L}^{T}=N\left(\widetilde{B}, \widetilde{W}^{3}, \widetilde{H}_{1}^{0}, \widetilde{H}_{2}^{0}\right)_{L}^{T}
$$

The mass eigenvalues $m_{\widetilde{\chi}_{i}^{0}}(i=1,2,3,4)$ in $\mathcal{M}_{\text {diag }}$ can be chosen positive by a suitable definition of the unitary matrix $N$.

The chargino mass matrix in the $\left(\widetilde{W}^{-}, \widetilde{H}^{-}\right)$basis is [25]

$$
\mathcal{M}_{C}=\left(\begin{array}{cc}
M_{2} & \sqrt{2} m_{W} \cos \beta \\
\sqrt{2} m_{W} \sin \beta & \mu
\end{array}\right)
$$

which is diagonalized by two unitary matrices ${ }^{3}$ through $U_{R} \mathcal{M}_{C} U_{L}^{\dagger}=\operatorname{diag}\left(m_{\widetilde{\chi}_{1}^{ \pm}}, m_{\widetilde{\chi}_{2}^{ \pm}}\right)$. The unitary matrices $U_{L}$ and $U_{R}$ can be parameterized in the following way [25]:

$$
U_{L}=\left(\begin{array}{cc}
\cos \phi_{L} & \mathrm{e}^{-i \beta_{L}} \sin \phi_{L} \\
-\mathrm{e}^{i \beta_{L}} \sin \phi_{L} & \cos \phi_{L}
\end{array}\right), \quad U_{R}=\left(\begin{array}{cc}
\mathrm{e}^{i \gamma_{1}} \cos \phi_{R} & \mathrm{e}^{i\left(\gamma_{1}-\beta_{R}\right)} \sin \phi_{R} \\
-\mathrm{e}^{i\left(\gamma_{2}+\beta_{R}\right)} \sin \phi_{R} & \mathrm{e}^{i \gamma_{2}} \cos \phi_{R}
\end{array}\right) \text {. }
$$

In CP-violating theories, the mass parameters are complex. Since $M_{2}$ can be taken real and positive by rephasing the fields suitably, the split SUSY scenario allows only two CPviolating phases of $M_{1}$ and $\mu$ :

$$
M_{1}=\left|M_{1}\right| \mathrm{e}^{i \Phi_{1}} \quad \text { and } \quad \mu=|\mu| \mathrm{e}^{i \Phi_{\mu}} \quad\left(0 \leq \Phi_{1}, \Phi_{\mu}<2 \pi\right)
$$

The universal gaugino mass relation at the GUT scale implies at the weak scale

$$
\left|M_{1}\right|=\frac{5}{3} \tan ^{2} \theta_{W} M_{2} \simeq 0.502 M_{2}
$$

The five underlying SUSY parameters $\left\{\left|M_{1}\right|, \Phi_{1},|\mu|, \Phi_{\mu} ; \tan \beta\right\}$ determine the mass spectrum and couplings of the neutralinos and charginos.

The interaction Lagrangian relevant for the production and decay of neutralinos and

\footnotetext{
${ }^{3} U_{L}$ and $U_{R}$ are related to $U$ and $V$ in Haber-Kane [26] notation by $V=U_{R}^{*}$ and $U=U_{L}$.
} 
charginos are expressed in 4-component form as

$$
\begin{aligned}
\mathcal{L}= & e \sum_{i} \overline{\widetilde{\chi}_{i}^{-}} \gamma^{\mu} \widetilde{\chi}_{i}^{-} A_{\mu}-g_{Z} \sum_{\alpha, i, j} Q_{\alpha i j}^{\mathrm{ccZ}} \overline{\widetilde{\chi}_{i}^{-}} \gamma^{\mu} P_{\alpha} \widetilde{\chi}_{j}^{-} Z_{\mu}-g_{Z} \sum_{\alpha, i, j} Q_{\alpha i j}^{\mathrm{nnZ}} \overline{\widetilde{\chi}_{i}^{0}} \gamma^{\mu} P_{\alpha} \widetilde{\chi}_{j}^{0} Z_{\mu} \\
& -g \sum_{i} S_{i}^{\mathrm{nnh}} \overline{\widetilde{\chi}_{i}^{0}} \widetilde{\chi}_{i}^{0} h^{0}-g \sum_{i<j} Q_{\alpha i j}^{\mathrm{nnh}} \overline{\widetilde{\chi}_{i}^{0}} P_{\alpha} \widetilde{\chi}_{j}^{0} h^{0} \\
& -g \sum_{\alpha, i, j} Q_{\alpha i j}^{\mathrm{cnW}} \overline{\widetilde{\chi}_{i}^{-}} \gamma^{\mu} P_{\alpha} \widetilde{\chi}_{j}^{0} W_{\mu}+H . c . \\
& -g \sum_{\alpha, i, j} Q_{\alpha i j}^{\mathrm{cnH}} \overline{\widetilde{\chi}_{i}^{-}} P_{\alpha} \widetilde{\chi}_{j}^{0} H^{-}-g \sum_{\alpha, i, j} Q_{\alpha i j}^{\mathrm{cnG}} \overline{\widetilde{\chi}_{i}^{-}} P_{\alpha} \widetilde{\chi}_{j}^{0} G^{-}+H . c .
\end{aligned}
$$

where $\alpha=L, R, P_{R, L}=\left(1 \pm \gamma^{5}\right) / 2, i, j=1,2$ for the chargino and $i, j=1, \cdots 4$ for the neutralino. ${ }^{4}$ Here $H^{-}$is the charged Higgs boson and $G^{-}$is the charged Goldstone boson. In the non-linear $R_{\xi}$ gauge used in the calculation of the radiative decay, the $G^{ \pm}$has the mass of $m_{W}$, of which the contribution is important. The detailed expressions for various couplings $Q_{\alpha i j}$ are listed in Appendix.

\section{DECAYS OF NEUTRALINOS AND CHARGINOS}

In general, the production cross section of gaugino-pair in split SUSY is smaller than the case with light sfermions. It brings a challenge to experimental search of gauginos. At the LHC, the gluino-pair production is dominated by the $g g$-initiated subprocess, which stays the same as in the MSSM. (Though the $q \bar{q}$-initiated subprocess changes, it is very small compared with $g g$-initiated one.) On the other hand, the detection is very different. Gluinos so produced would be detected as massive stable charged particles [14, 15, 16] or as a gluinonium [17]. Even though the detection of heavy meta-stable gluinos alone can strongly support the split SUSY scenario, it cannot provide any more information on the fundamental SUSY parameters involved in the neutralino and chargino sectors. Even with small production rates, the decays of neutralinos and charginos in split SUSY are to be studied in detail.

In split SUSY, the chargino decay is very simple. It would decay into $\widetilde{\chi}_{1}^{0} W^{(*)}$ giving rise to a single charged lepton or two jets plus missing energy. On the other hand, the heavier neutralino $\widetilde{\chi}_{j}^{0}$ have a few more decay modes.

\footnotetext{
${ }^{4}$ Note that $\tilde{\chi}^{-}$is considered as a particle, contrary to the Haber and Kane notation.
} 
- $\widetilde{\chi}_{j}^{0} \rightarrow \widetilde{\chi}_{i}^{0} Z^{(*)} \rightarrow \widetilde{\chi}_{i}^{0}(j j / \ell \bar{\ell})$, giving rise to a couple of jets or charged leptons plus missing energy.

- $\widetilde{\chi}_{j}^{0} \rightarrow \widetilde{\chi}_{i}^{0} h^{(*)} \rightarrow \widetilde{\chi}_{i}^{0}\left(b \bar{b} / W W^{*}\right)$. If the Higgs boson is lighter than about $125 \mathrm{GeV}$, it decays dominantly into $b \bar{b}$. With $m_{h} \geq 130 \mathrm{GeV}$ the $W W^{*}$ mode becomes important, which is possible because the Higgs mass can be substantially larger in split SUSY [5, 8, 9,10$]$.

- $\widetilde{\chi}_{j}^{0} \rightarrow \widetilde{\chi}_{i}^{ \pm} W^{\mp(*)} \rightarrow \widetilde{\chi}_{i}^{ \pm} f \bar{f}^{\prime}$. This mode happens when the heavier neutralino is heavier than the lightest chargino, especially, in the region where the $\mu$ parameter is smaller than $M_{2}$.

- $\widetilde{\chi}_{j}^{0} \rightarrow \widetilde{\chi}_{i}^{0} \gamma[27]$. This mode goes through the chargino- $W$ loop, as the loops involving sfermions are highly suppressed. This decay mode gives a single photon and missing energy.

The expressions for the decay rates of first three decay modes are greatly simplified in split SUSY. We give the formulas for various decays of the neutralino $\widetilde{\chi}_{j}^{0}$ into $\widetilde{\chi}_{i}^{0}$. Note that for decays of $\widetilde{\chi}_{3,4}^{0}$ they may go through $\widetilde{\chi}_{2}^{0}$ before they end up in $\widetilde{\chi}_{1}^{0}$.

\section{A. Two body decays of $\widetilde{\chi}_{j}^{0} \rightarrow \widetilde{\chi}_{i}^{0} Z, \widetilde{\chi}_{i}^{0} h^{0}, \widetilde{\chi}_{i}^{ \pm} W^{\mp}$}

For simplicity we introduce some short-hand notation of

$$
\mu_{i j}=\left(\frac{m_{\tilde{\chi}_{i}^{0( \pm)}}}{m_{\tilde{\chi}_{j}^{0}}}\right)^{2}, \quad \mu_{X j}=\left(\frac{m_{X}}{m_{\tilde{\chi}_{j}^{0}}}\right)^{2},
$$

where $X=Z, h^{0}, W^{ \pm}$. If $m_{\widetilde{\chi}_{j}^{0}}>m_{\widetilde{\chi}_{i}^{\left({ }^{( \pm)}\right.}}+m_{X}, \widetilde{\chi}_{j}^{0}$ decays into a lighter neutralino or chargino associated with the $X$ boson. The total decay rate is then

$$
\Gamma\left(\widetilde{\chi}_{j}^{0} \rightarrow \widetilde{\chi}_{i}^{0( \pm)} X\right)=\frac{\lambda^{1 / 2}\left(1, \mu_{i j}, \mu_{X j}\right)}{16 \pi m_{\widetilde{\chi}_{j}^{0}}} \overline{|\mathcal{M}|^{2}}
$$


where $\lambda(a, b, c)=a^{2}+b^{2}+c^{2}-2 a b-2 b c-2 c a$ and $\overline{|\mathcal{M}|^{2}}$ is the spin-average amplitude squared. For each decay mode $\overline{|\mathcal{M}|^{2}}$ is

$$
\begin{aligned}
\overline{|\mathcal{M}|^{2}}\left(\widetilde{\chi}_{j}^{0} \rightarrow \widetilde{\chi}_{i}^{0} Z\right)=\frac{g_{Z}^{2} m_{\widetilde{\chi}_{j}^{0}}^{2}}{2}\left[\left(\left|Q_{R i j}^{\mathrm{nnZ}}\right|^{2}+\left|Q_{L i j}^{\mathrm{nnZ}}\right|^{2}\right)\left\{\frac{\left(1-\mu_{i j}\right)^{2}}{\mu_{Z j}}+1+\mu_{i j}-2 \mu_{Z j}\right\}\right. & \left.-12 \sqrt{\mu_{i j}} \Re e\left(Q_{R i j}^{\mathrm{nnZ}} Q_{L i j}^{\mathrm{nnZ} *}\right)\right], \\
\overline{|\mathcal{M}|^{2}}\left(\widetilde{\chi}_{j}^{0} \rightarrow \widetilde{\chi}_{i}^{0} h^{0}\right)= & \frac{g^{2} m_{\tilde{\chi}_{j}^{0}}^{2}}{2}\left[\left(\left|Q_{R i j}^{\mathrm{nnh}}\right|^{2}+\left|Q_{L i j}^{\mathrm{nnh}}\right|^{2}\right)\left(1+\mu_{i j}-\mu_{h j}\right)\right. \\
& \left.+4 \sqrt{\mu_{i j}} \Re e\left(Q_{R i j}^{\mathrm{nnh}} Q_{L i j}^{\mathrm{nnh} *}\right)\right], \\
\overline{|\mathcal{M}|^{2}}\left(\widetilde{\chi}_{j}^{0} \rightarrow \widetilde{\chi}_{i}^{-} W^{+}\right)= & \overline{|\mathcal{M}|^{2}}\left(\widetilde{\chi}_{j}^{0} \rightarrow \widetilde{\chi}_{i}^{+} W^{-}\right) \\
= & \frac{g^{2} m_{\widetilde{\chi}_{j}^{0}}^{2}}{2}\left[\left(\left|Q_{L 1 j}^{\mathrm{cnW}}\right|^{2}+\left|Q_{R 1 j}^{\mathrm{cnW}}\right|^{2}\right)\left(\frac{\left(1-\mu_{i j}\right)^{2}}{\mu_{W j}}+1+\mu_{i j}-2 \mu_{W j}\right)\right. \\
& \left.-12 \sqrt{\mu_{i j}} \Re e\left(Q_{L 1 j}^{\mathrm{cnW}} Q_{R 1 j}^{\mathrm{cnW} *}\right)\right] .
\end{aligned}
$$

\section{B. Three body decays of $\widetilde{\chi}_{j}^{0} \rightarrow \widetilde{\chi}_{i}^{0, \pm} f \bar{f}^{(\prime)}$}

If $m_{\widetilde{\chi}_{j}^{0}}<m_{\widetilde{\chi}_{i}^{0( \pm)}}+m_{X}$, the decay will proceed into $\widetilde{\chi}_{i}^{0( \pm)} f \bar{f}^{\left({ }^{\prime}\right)}$ via a virtual $Z^{*}, W^{*}$ or $h^{*}$. The differential decay width is given by

$$
\frac{d \Gamma}{d x_{f} d x_{\bar{f}}}=\frac{N_{C} m_{\widetilde{\chi}_{j}^{0}}}{256 \pi^{3}} \overline{|\mathcal{M}|^{2}},
$$

where $N_{C}$ is the color factor of the fermion $f$ and the kinematic variables are defined in the rest frame of $\widetilde{\chi}_{j}^{0}$ by

$$
x_{f}=\frac{2 E_{f}}{m_{\widetilde{\chi}_{j}^{0}}}, \quad x_{\bar{f}}=\frac{2 E_{\bar{f}}}{m_{\widetilde{\chi}_{j}^{0}}}, \quad x_{i}=2-x_{f}-x_{\bar{f}} .
$$

The integration range of $x_{f}$ and $x_{\bar{f}}$ are, with the definition of $\mu_{f(\bar{f})} \equiv m_{f(\bar{f})}^{2} / m_{\tilde{\chi}_{j}^{0}}^{2}$,

$$
\begin{aligned}
2 \sqrt{\mu_{f}} & \leq x_{f} \leq 1+\mu_{f}-\mu_{\bar{f}}-\mu_{i j}-2 \sqrt{\mu_{\bar{f}} \mu_{i j}} \\
x_{\bar{f}_{(-)}} & \leq x_{\bar{f}} \leq x_{\bar{f}_{(+)}}
\end{aligned}
$$

where

$$
\begin{aligned}
x_{\bar{f}_{( \pm)}}=\frac{1}{2\left(1-x_{f}+\mu_{f}\right)}[ & \left(2-x_{f}\right)\left(1+\mu_{f}+\mu_{\bar{f}}-\mu_{i j}-x_{f}\right) \\
& \left. \pm \sqrt{\left(x_{f}^{2}-4 \mu_{f}\right) \lambda\left(1+\mu_{f}-x_{f}, \mu_{\bar{f}}, \mu_{i j}\right)}\right] .
\end{aligned}
$$


The spin-average amplitude squared for each decay mode is

$$
\begin{aligned}
\overline{|\mathcal{M}|^{2}}\left(\widetilde{\chi}_{j}^{0} \rightarrow \widetilde{\chi}_{i}^{0} Z^{*} \rightarrow \widetilde{\chi}_{i}^{0} f \bar{f}\right) \\
=g_{z}^{4}\left(\left|g_{L}^{f}\right|^{2}+\left|g_{R}^{f}\right|^{2}\right) \hat{d}_{Z}^{2}\left[\left\{\left(x_{f}+x_{\bar{f}}\right)\left(1-\mu_{i j}\right)-x_{f}^{2}-x_{\bar{f}}^{2}\right\}\left(\left|Q_{L i j}^{\mathrm{nnZ}}\right|^{2}+\left|Q_{R i j}^{\mathrm{nnZ}}\right|^{2}\right)\right. \\
\left.\quad-4 \sqrt{\mu_{i j}}\left(1+\mu_{i j}-x_{i}\right) \Re e\left(Q_{L i j}^{\mathrm{nnZ}} Q_{R i j}^{\mathrm{nnZ} *}\right)\right] \\
\overline{|\mathcal{M}|^{2}}\left(\widetilde{\chi}_{j}^{0} \rightarrow \widetilde{\chi}_{i}^{0} h^{*} \rightarrow \widetilde{\chi}_{i}^{0} b \bar{b}\right) \\
=\frac{g^{4} m_{b}^{2} \sin ^{2} \alpha}{4 m_{W}^{2} \cos ^{2} \beta} \hat{d}_{h}^{2}\left(1+\mu_{i j}-x_{i}-2 \mu_{b}\right)\left[x_{i}\left(\left|Q_{L i j}^{\mathrm{nnh}}\right|^{2}+\left|Q_{R i j}^{\mathrm{nnh}}\right|^{2}\right)+4 \sqrt{\mu_{i j}} \Re e\left(Q_{L i j}^{\mathrm{nnh}} Q_{R i j}^{\mathrm{nnn} *}\right)\right] \\
\overline{|\mathcal{M}|^{2}}\left(\widetilde{\chi}_{j}^{0} \rightarrow \widetilde{\chi}_{i}^{-} W^{*} \rightarrow \widetilde{\chi}_{i}^{-} f \bar{f}^{\prime}\right) \\
=g^{4} \hat{d}_{W}^{2}\left[x_{f}\left(1-\mu_{i j}-x_{f}\right)\left|Q_{L i j}^{\mathrm{cnW}}\right|^{2}+x_{\bar{f}}\left(1-\mu_{i j}-x_{\bar{f}}\right)\left|Q_{R i j}^{\mathrm{cnW}}\right|^{2}\right. \\
\left.\quad-2 \sqrt{\mu_{i j}}\left(1+\mu_{i}-x_{i}\right) \Re e\left(Q_{L i j}^{\mathrm{cnW}} Q_{R i j}^{\mathrm{cnW} *}\right)\right]
\end{aligned}
$$

where the propagator factor $\hat{d}_{X}$, with $X=Z, h^{0}, W$, is

$$
\hat{d}_{X}=\frac{m_{\widetilde{\chi}_{j}^{0}}^{2}}{\left(p_{f}+p_{\bar{f}}\right)^{2}-m_{X}^{2}}=\frac{1}{1+\mu_{i j}-x_{i}-\mu_{X j}} .
$$

Here the chiral couplings of the fermion $f$ to the $Z$ boson are given by

$$
g_{R}^{f}=-s_{W}^{2} Q_{f}, \quad g_{L}^{f}=\left(T_{3}^{f}\right)_{L}-s_{W}^{2} Q_{f},
$$

where $\left(T_{3}^{f}\right)_{L}$ is the third component of the isospin and $Q_{f}$ is the electric charge.

\section{Radiative decay}

In split SUSY, the radiative decay of neutralino, $\widetilde{\chi}_{j}^{0} \rightarrow \widetilde{\chi}_{i}^{0} \gamma$, may have a substantial branching fraction as the other decay channels are limited to $\widetilde{\chi}_{j}^{0} \rightarrow \widetilde{\chi}_{i}^{0} Z^{(*)}, \widetilde{\chi}_{j}^{0} \rightarrow \widetilde{\chi}_{i}^{0} h^{(*)}$, and $\tilde{\chi}_{j}^{0} \rightarrow \widetilde{\chi}_{i}^{ \pm} W^{\mp(*)}$. As discussed in Ref. [27], the radiative neutralino decay proceeds through triangle diagrams mediated by the $f \tilde{f}, \tilde{\chi}^{ \pm} W^{\mp}, \widetilde{\chi}^{ \pm} H^{\mp}$, and $\tilde{\chi}^{ \pm} G^{\mp}$ loops in the non-linear $R_{\xi}$ gauge where the charged Goldstone boson $G^{ \pm}$has the same mass as $m_{W}$. In split SUSY,

only $W^{ \pm}$- and $G^{ \pm}$-mediated diagrams contribute. We expand the results of Ref. 27] into the $\mathrm{CP}$-violating case, and examine whether $\mathrm{CP}$-violating phases can enhance the radiative decay rate of neutralinos.

For the decay of

$$
\tilde{\chi}_{j}^{0}(p) \rightarrow \widetilde{\chi}_{i}^{0}\left(k_{1}\right)+\gamma\left(k_{2}\right),
$$


the matrix element is, in general, given by

$$
\mathcal{M}=\frac{1}{m_{\tilde{\chi}_{j}^{0}}} \bar{u}\left(k_{1}\right)\left(g_{v}+g_{a} \gamma_{5}\right) \not k_{2} \notin^{*} u(p),
$$

where $\epsilon$ denotes the polarization 4-vector of the photon, and the radiative decay width of $\widetilde{\chi}_{j}^{0}$ can be easily calculated as

$$
\Gamma\left(\widetilde{\chi}_{j}^{0} \rightarrow \widetilde{\chi}_{i}^{0} \gamma\right)=\left(\left|g_{v}\right|^{2}+\left|g_{a}\right|^{2}\right) \frac{\left(m_{\widetilde{\chi}_{j}^{0}}^{2}-m_{\widetilde{\chi}_{i}^{0}}^{2}\right)^{3}}{8 \pi m_{\widetilde{\chi}_{j}^{0}}^{5}} .
$$

In the notation of Ref. [27], the coupling of the incoming neutralino $\tilde{\chi}_{j}^{0}$ to the particles in the loop is denoted by

$$
F^{X}=f_{L}^{X} P_{L}+f_{R}^{X} P_{R}
$$

and the coupling of the outgoing neutralino $\widetilde{\chi}_{i}^{0}$ by

$$
G^{X}=g_{L}^{X} P_{L}+g_{R}^{X} P_{R}
$$

where $X=W^{ \pm}, G^{ \pm}$and $-i g \gamma^{\mu}(-i g)$ is omitted for the $W^{ \pm}\left(G^{ \pm}\right)$loop respectively. Explicitly we have

$$
\begin{gathered}
f_{\alpha}^{W}=Q_{\alpha k j}^{\mathrm{cnW}}, \quad g_{\alpha}^{W}=\left(Q_{\alpha k i}^{\mathrm{cnW}}\right)^{*}, \\
f_{\alpha}^{G}=Q_{\alpha k j}^{\mathrm{cnG}}, \quad g_{\alpha}^{G}=\left(Q_{\bar{\alpha} k i}^{\mathrm{cnG}}\right)^{*},
\end{gathered}
$$

where $\bar{\alpha}=R(L)$ for $\alpha=L(R)$. The helicity amplitude in the CP-violating cases is

$$
\mathcal{M}=\sum_{X=W, G} \frac{1}{m_{\tilde{\chi}_{j}^{0}}} \frac{e g^{2}}{8 \pi^{2}} \bar{u}\left(k_{1}\right)\left(g_{V}^{X}+g_{A}^{X} \gamma_{5}\right) k_{2} \ddagger^{*} u(p),
$$


where $g_{V, A}^{X}$ are given by

$$
\begin{aligned}
& g_{V}^{W}=i \sum_{k=1,2}\left[\Im m\left(g_{L}^{W} f_{L}^{W}+g_{R}^{W} f_{R}^{W}\right)\left\{m_{\widetilde{\chi}_{j}^{0}}^{2}\left(I_{2}-J-K\right)-m_{\widetilde{\chi}_{j}^{0}} m_{\widetilde{\chi}_{i}^{0}}(J-K)\right\}\right. \\
& \left.+2 m_{\widetilde{\chi}_{j}^{0}} m_{\widetilde{\chi}_{k}^{ \pm}} \Im m\left(g_{L}^{W} f_{R}^{W}+g_{R}^{W} f_{L}^{W}\right) J\right], \\
& g_{A}^{W}=\sum_{k=1,2}\left[\Re \mathrm{e}\left(g_{L}^{W} f_{L}^{W}-g_{R}^{W} f_{R}^{W}\right)\left\{m_{\widetilde{\chi}_{j}^{0}}^{2}\left(I_{2}-J-K\right)+m_{\widetilde{\chi}_{j}^{0}} m_{\widetilde{\chi}_{i}^{0}}(J-K)\right\}\right. \\
& \left.+2 m_{\widetilde{\chi}_{j}^{0}} m_{\widetilde{\chi}_{k}^{ \pm}} \Re \mathrm{e}\left(g_{L}^{W} f_{R}^{W}-g_{R}^{W} f_{L}^{W}\right) J\right], \\
& g_{V}^{G}=\frac{i}{4} \sum_{k=1,2}\left[\Im \mathrm{m}\left(g_{L}^{G} f_{R}^{G}+g_{R}^{G} f_{L}^{G}\right)\left\{m_{\widetilde{\chi}_{j}^{0}}^{2}\left(I_{2}-K\right)+m_{\tilde{\chi}_{j}^{0}} m_{\widetilde{\chi}_{i}^{0}} K\right\}\right. \\
& \left.+m_{\widetilde{\chi}_{j}^{0}} m_{\widetilde{\chi}_{k}^{ \pm}} \Im \mathrm{m}\left(g_{L}^{G} f_{L}^{G}+g_{R}^{G} f_{R}^{G}\right) I\right], \\
& g_{A}^{G}=-\frac{1}{4} \sum_{k=1,2}\left[\Re \mathrm{e}\left(g_{L}^{G} f_{R}^{G}-g_{R}^{G} f_{L}^{G}\right)\left\{m_{\widetilde{\chi}_{j}^{0}}^{2}\left(I_{2}-K\right)-m_{\widetilde{\chi}_{j}^{0}} m_{\widetilde{\chi}_{i}^{0}} K\right\}\right. \\
& \left.+m_{\widetilde{\chi}_{j}^{0}} m_{\widetilde{\chi}_{k}^{ \pm}} \Re \mathrm{e}\left(g_{L} f_{L}-g_{R} f_{R}\right) I\right] .
\end{aligned}
$$

The radiative decay width is

$$
\Gamma=\frac{\alpha^{3}}{8 \pi^{2} \sin \theta_{W}^{4}}\left(\left|g_{V}\right|^{2}+\left|g_{A}\right|^{2}\right) \frac{\left(m_{\widetilde{\chi}_{j}^{0}}^{2}-m_{\widetilde{\chi}_{i}^{0}}^{2}\right)^{3}}{m_{\widetilde{\chi}_{j}^{0}}^{5}},
$$

where $g_{V, A} \equiv \sum_{X} g_{V, A}^{X}$. The loop integrals $I, I_{2}, J, K$ are the same as in Ref. [27].

\section{Numerical Results of neutralino decay rates}

Focusing on the parameter space of $|\mu|$ compatible with $M_{1}$, we consider $|\mu| \in[160,230]$ $\mathrm{GeV}$ for $M_{1}=200 \mathrm{GeV}$. With the gaugino mass unification condition of $\left|M_{1}\right| \simeq 0.502 M_{2}$, the mass hierarchy among the neutralinos and charginos is then $m_{\widetilde{\chi}_{1}^{0}}<m_{\widetilde{\chi}_{1}^{ \pm}}<m_{\widetilde{\chi}_{2}^{0}}<m_{\widetilde{\chi}_{3}^{0}}$.

First, we compute the partial decay widths of $\widetilde{\chi}_{2}^{0}$ and $\widetilde{\chi}_{3}^{0}$ in the CP-conserving case $\left(\Phi_{1}=\Phi_{\mu}=0\right)$ in Figs. 1 and 2 respectively. Considering the trigger strategy at the LHC, we separate the electron and muon signal from other fermion signals. The notation of $\sum f \bar{f}^{\left({ }^{\prime}\right)}$ includes all appropriate fermions $f, f^{\prime}$ except for $e^{ \pm}$and $\mu^{ \pm}$. In the decay of $\widetilde{\chi}_{2}^{0}$, the majority comes from $\widetilde{\chi}_{2}^{0} \rightarrow \widetilde{\chi}_{1}^{0} f \bar{f}$ via an on-shell or a virtual $Z$. It is followed by $\tilde{\chi}_{2}^{0} \rightarrow \tilde{\chi}^{ \pm} f \bar{f}^{\prime}$ via an on-shell or a virtual $W$. The radiative decay width is down by 3 orders of magnitude from $\widetilde{\chi}_{2}^{0} \rightarrow \widetilde{\chi}_{1}^{0} Z^{(*)} \rightarrow \widetilde{\chi}_{1}^{0} f \bar{f}$ in the range of $\mu$ that we are interested in. Figure 2 shows the partial widths of $\widetilde{\chi}_{3}^{0}$. A substantial mass difference between $m_{\widetilde{\chi}_{3}^{0}}$ and $m_{\widetilde{\chi}_{1}^{-}}$for 


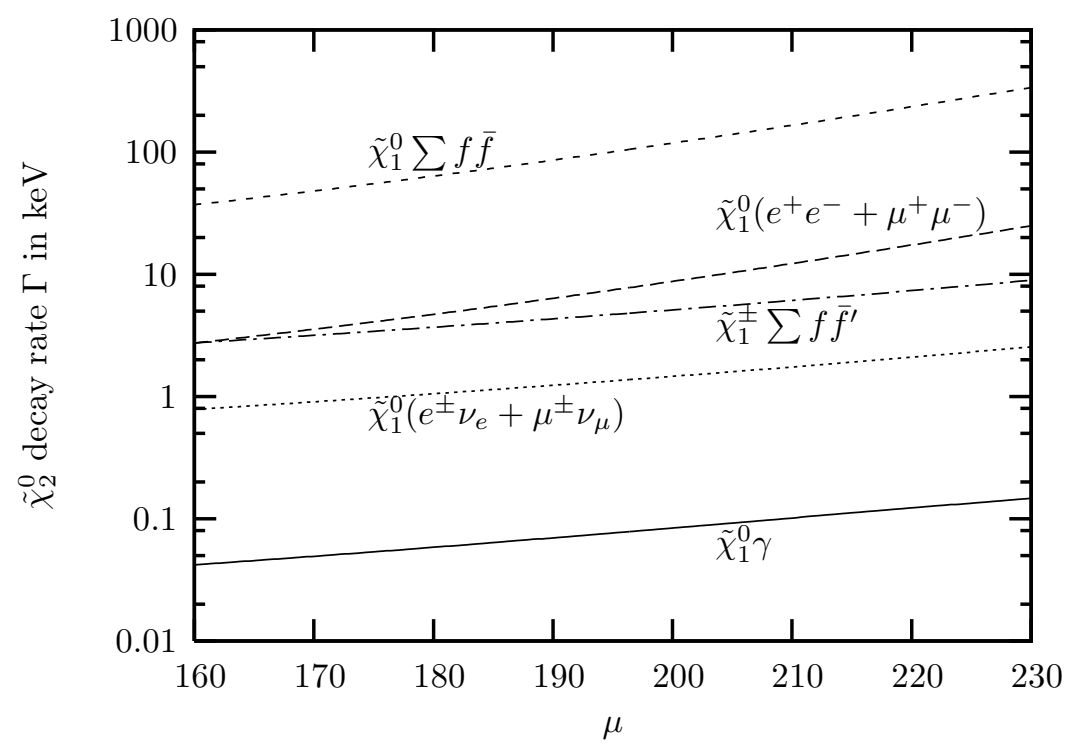

FIG. 1: Partial decay widths of $\widetilde{\chi}_{2}^{0}$ for $M_{1}=200 \mathrm{GeV}$ and $\tan \beta=10$. All the $\mathrm{CP}$ violating phases are set to zero. $\sum f \bar{f}^{(\prime)}$ includes all possible SM fermion pairs except for $e^{ \pm}$and $\mu^{ \pm}$involving pairs.

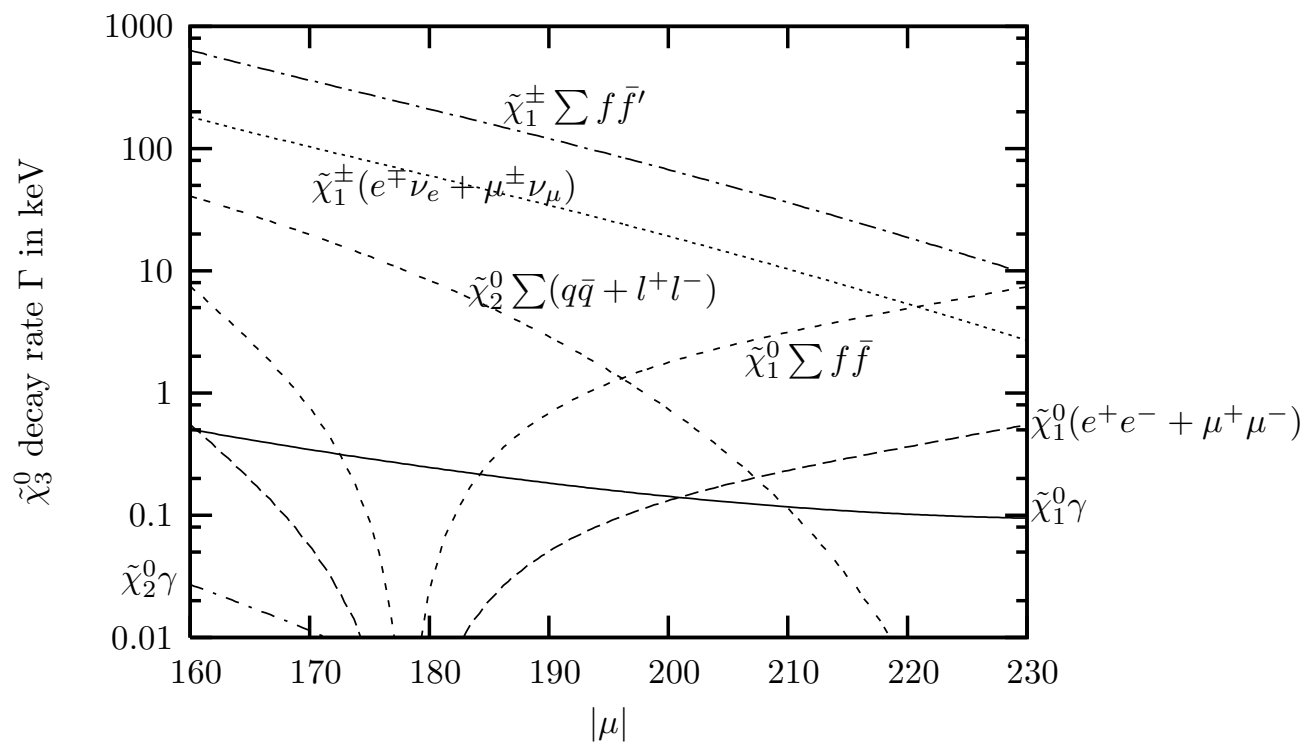

FIG. 2: Partial decay widths of $\widetilde{\chi}_{3}^{0}$ for $M_{1}=200 \mathrm{GeV}$ and $\tan \beta=10$. All the $\mathrm{CP}$ violating phases are set to zero.

$|\mu|$ compatible with $M_{1}$ leads to the dominant decay mode into $\tilde{\chi}^{ \pm} f \bar{f}^{\prime}$ via an on-shell or a virtual $W$. It is followed by $\widetilde{\chi}_{3}^{0} \rightarrow \widetilde{\chi}_{2}^{0} Z^{(*)} \rightarrow \widetilde{\chi}_{2}^{0} f \bar{f}$, which drops off quickly when $\mu$ increases. A special dip (around $|\mu| \simeq 178 \mathrm{GeV}$ with $M_{1}=200 \mathrm{GeV}$ ) shows up in the decay width of $\widetilde{\chi}_{3}^{0} \rightarrow \widetilde{\chi}_{1}^{0} Z^{(*)} \rightarrow \widetilde{\chi}_{1}^{0} f \bar{f}$. This is because of a cancellation in the factor $\left(N_{13} N_{33}^{*}-N_{14} N_{34}^{*}\right)$ of 


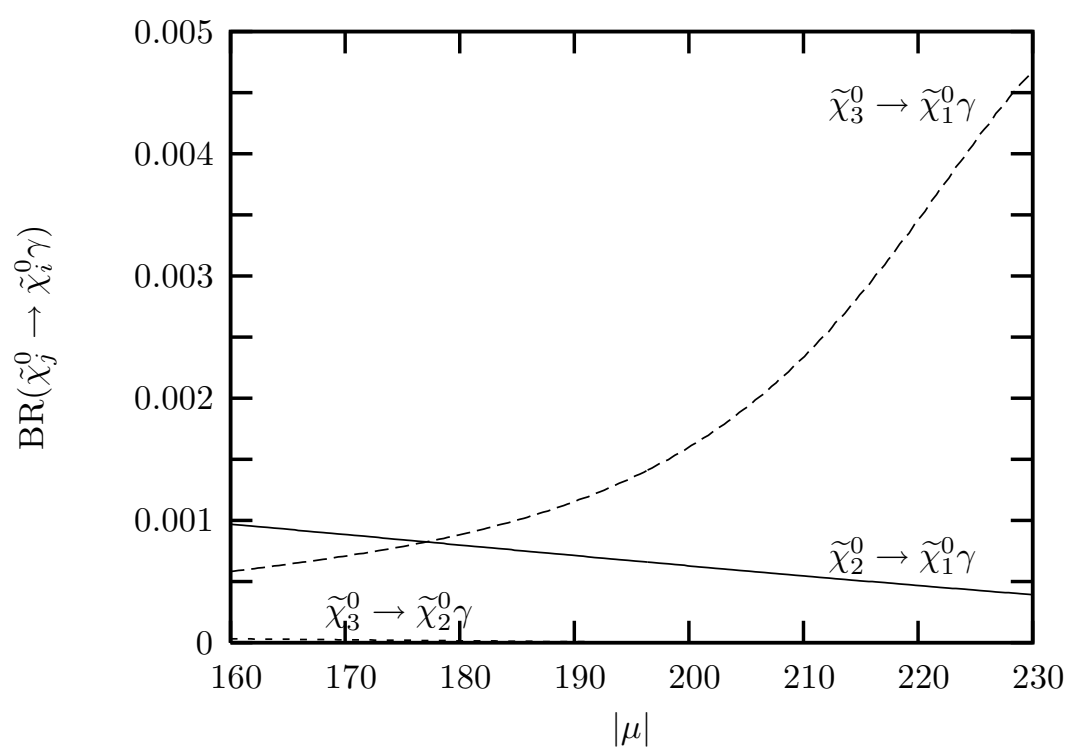

FIG. 3: Radiative decay branching ratios for $\widetilde{\chi}_{2}^{0}$ and $\widetilde{\chi}_{3}^{0}$ with $M_{1}=200 \mathrm{GeV}$ and $\tan \beta=10$. All the $\mathrm{CP}$ violating phases are set to zero.

the $\widetilde{\chi}_{3}^{0}-\widetilde{\chi}_{1}^{0}-Z$ coupling. The radiative decay width of $\widetilde{\chi}_{3}^{0} \rightarrow \widetilde{\chi}_{1}^{0} \gamma$ is relatively much larger than in the case of $\widetilde{\chi}_{2}^{0}$. This is the mode that we want to make use of in the collider study of $\widetilde{\chi}_{3}^{0}$ in split SUSY. Since the masses of $\widetilde{\chi}_{3}^{0}$ and $\widetilde{\chi}_{1}^{0}$ cannot be degenerate, the outgoing photon is quite energetic. A single-photon plus missing energy event search is possible provided that the branching ratio is large enough. We show the radiative decay branching ratios for $\widetilde{\chi}_{2}^{0} \rightarrow \widetilde{\chi}_{1}^{0} \gamma, \widetilde{\chi}_{3}^{0} \rightarrow \widetilde{\chi}_{1}^{0} \gamma$, and $\widetilde{\chi}_{3}^{0} \rightarrow \widetilde{\chi}_{2}^{0} \gamma$ in Fig. 目, The branching ratio for $\widetilde{\chi}_{3}^{0} \rightarrow \widetilde{\chi}_{2}^{0} \gamma$ is much smaller than the other two. With increasing $|\mu|, \operatorname{BR}\left(\widetilde{\chi}_{2}^{0} \rightarrow \widetilde{\chi}_{1}^{0} \gamma\right)$ moderately decreases while $\operatorname{BR}\left(\widetilde{\chi}_{3}^{0} \rightarrow \widetilde{\chi}_{1}^{0} \gamma\right)$ increases rather rapidly.

Finally, we examine the dependence of $\mathrm{CP}$-violating phases on the neutralino radiative decay. The effect of $\Phi_{1}$ is quite weak, as expected, since $M_{1}$ is involved only in the neutralino mass matrix. On the other hand, the $\Phi_{\mu}$ dependence is rather strong. The $\mu$ parameter affects both the neutralino and chargino sectors, and the $\widetilde{\chi}_{j}^{0}-\widetilde{\chi}_{i}^{ \pm}-W^{\mp}$ coupling in the loop. We compute the $\Phi_{\mu}$-dependence on the radiative branching ratios of $\widetilde{\chi}_{2}^{0}$ and $\widetilde{\chi}_{3}^{0}$ in Fig. 团

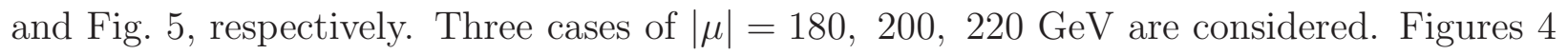
and 5 clearly show the CP-phase sensitivity of the neutralino radiative branching ratios. As can be seen in Fig. 5 the $\operatorname{BR}\left(\widetilde{\chi}_{3}^{0} \rightarrow \widetilde{\chi}_{1}^{0} \gamma\right)$ can be enhanced by a factor of about four with $|\mu|=220 \mathrm{GeV}$ at $\Phi_{\mu}=\pi$. In most cases, the maximum of radiative branching ratios occurs at $\Phi_{\mu}=\pi$, i.e., negative $\mu$. We also see the $\tan \beta$ dependence by plotting $\operatorname{BR}\left(\widetilde{\chi}_{3}^{0} \rightarrow \widetilde{\chi}_{1}^{0} \gamma\right)$ 


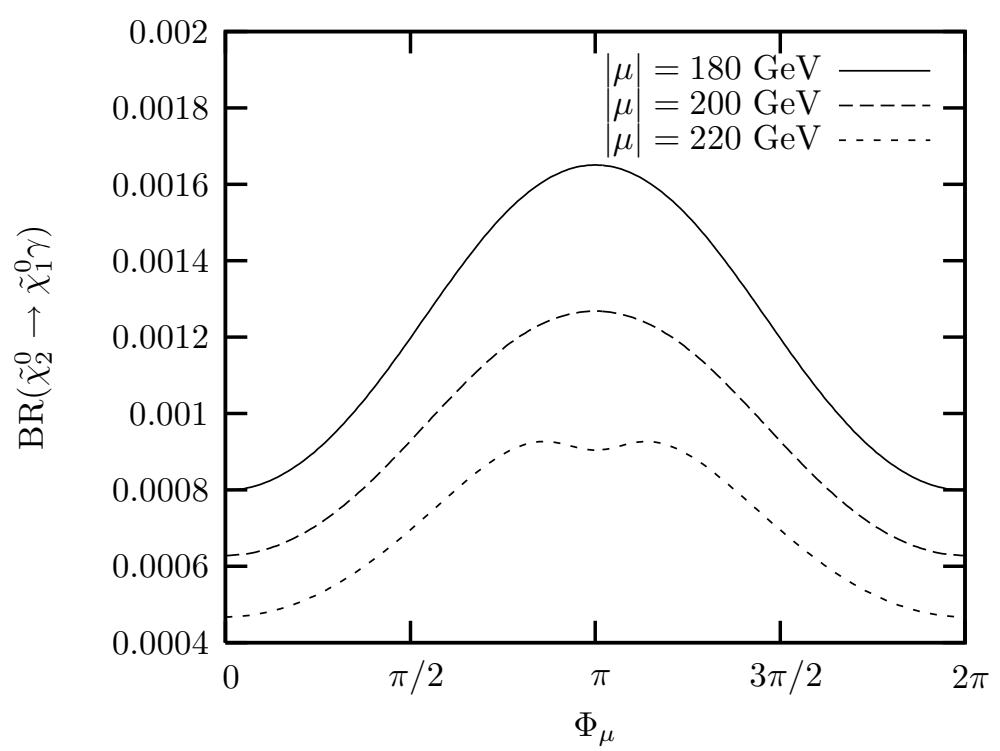

FIG. 4: Radiative decay branching ratio of $\widetilde{\chi}_{2}^{0} \rightarrow \widetilde{\chi}_{1}^{0} \gamma$ as a function of $\Phi_{\mu}$ (in unit of $\pi$ ) for $M_{1}=200 \mathrm{GeV}$ and $\tan \beta=10$. Three cases for $|\mu|=180,200,220 \mathrm{GeV}$ are shown.

for $\tan \beta=50$ and $|\mu|=220 \mathrm{GeV}$ in Fig. 5 . At $\Phi_{\mu}=0$, larger $\tan \beta$ enhances the radiative branching ratio by a factor of about two. However, at $\Phi_{\mu}=\pi$ where the radiative $\mathrm{BR}$ is maximized, $\tan \beta=50$ case has smaller $\mathrm{BR}$, about half of that with $\tan \beta=10$. In Fig. 6. we show the variation of the branching ratio versus negative $\mu$. It is now clear that at $\mu=-220 \mathrm{GeV}$, a branching ratio about $1.68 \%$ can be obtained for the radiative decay $\widetilde{\chi}_{3}^{0} \rightarrow \widetilde{\chi}_{1}^{0} \gamma$ with $\tan \beta=10$.

\section{PRODUCTION OF NEUTRALINOS AND CHARGINOS}

In split SUSY, gaugino-pair production goes through Drell-Yan-like process via $\gamma, Z$, or $W s$-channel exchange diagrams. Specifically, we study the production of

$$
q+\bar{q} \rightarrow \tilde{\chi}_{i}^{0}+\tilde{\chi}_{j}^{0}, \quad q+\bar{q} \rightarrow \tilde{\chi}_{i}^{-}+\tilde{\chi}_{j}^{+}, \quad q+\bar{q}^{\prime} \rightarrow \tilde{\chi}_{i}^{ \pm}+\tilde{\chi}_{j}^{0}
$$

For simplicity we introduce the following notation:

$$
\mu_{i \hat{s}}=\frac{m_{i}^{2}}{\hat{s}}, \quad D_{X}=\frac{\hat{s}}{\hat{s}-m_{X}^{2}+i m_{X} \Gamma_{X}},
$$




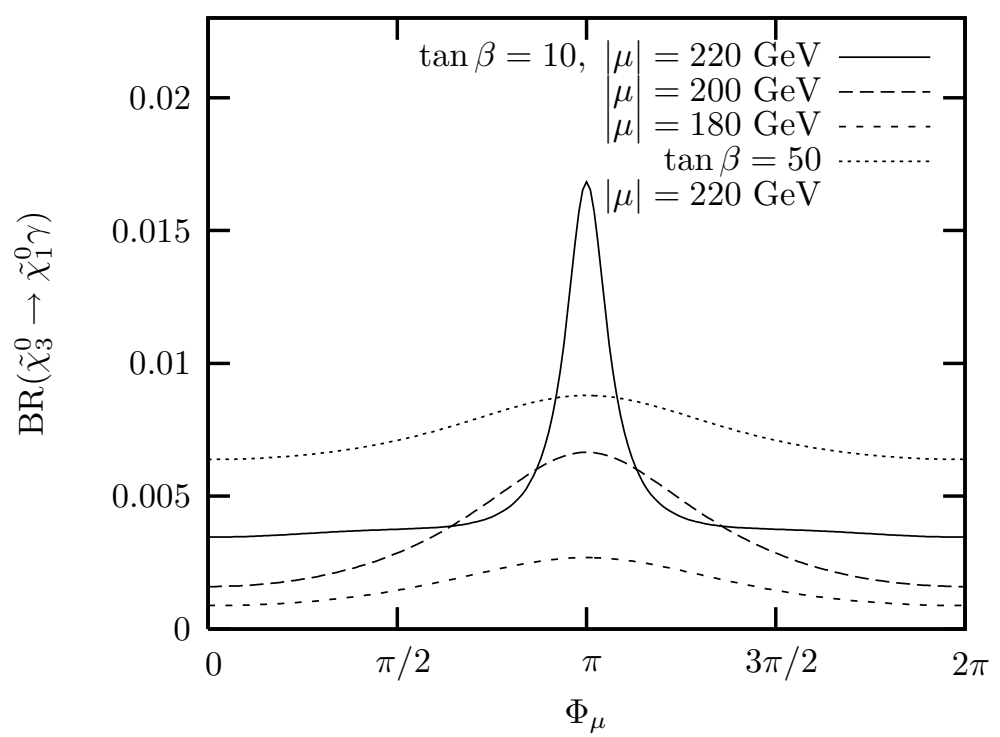

FIG. 5: Radiative decay branching ratio of $\widetilde{\chi}_{3}^{0} \rightarrow \widetilde{\chi}_{1}^{0} \gamma$ as a function of $\Phi_{\mu}$ (in unit of $\pi$ ) for $M_{1}=200 \mathrm{GeV}$ and $\tan \beta=10$. Three cases for $|\mu|=180,200,220 \mathrm{GeV}$ are shown. One additional line (dotted) of $\tan \beta=50$ and $|\mu|=220 \mathrm{GeV}$ is also shown.

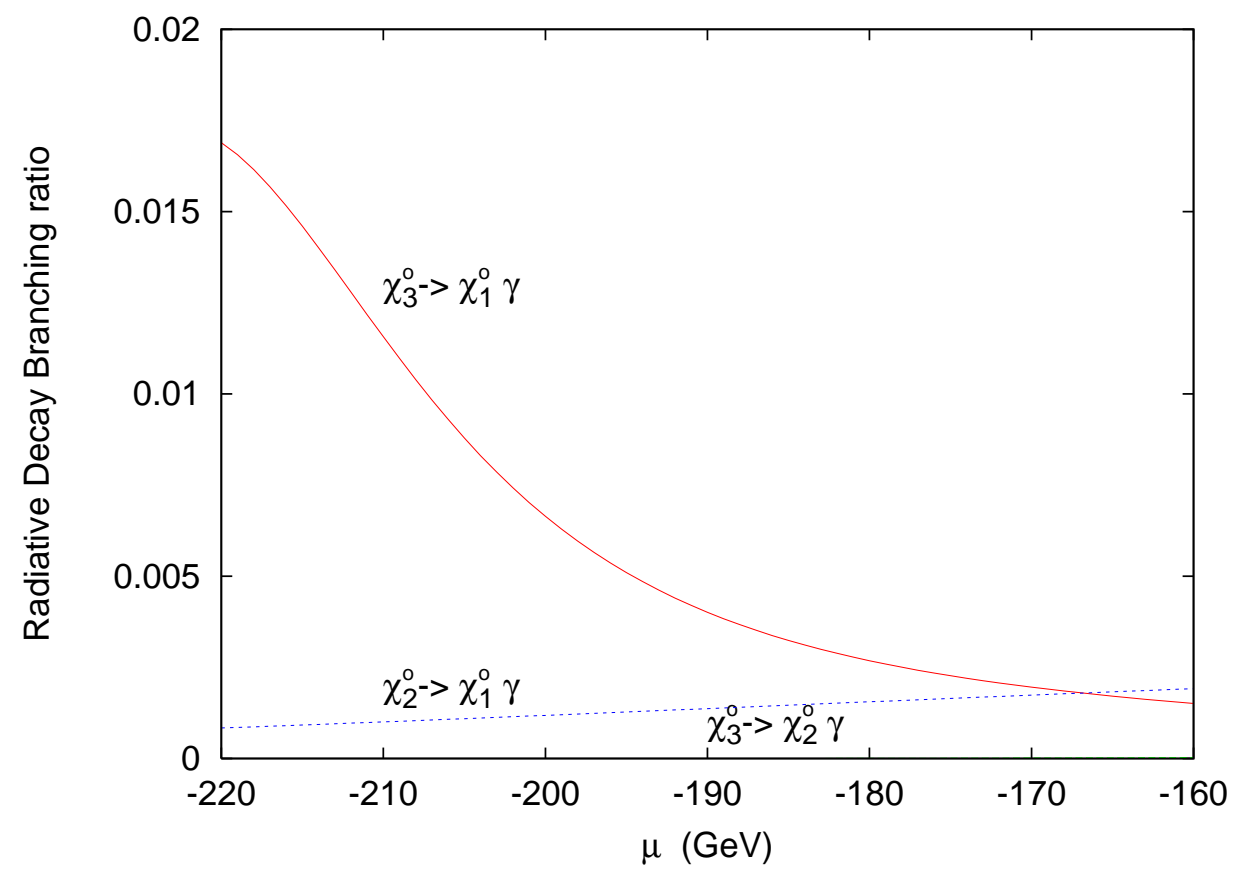

FIG. 6: Radiative decay branching ratios for $\widetilde{\chi}_{2}^{0}$ and $\widetilde{\chi}_{3}^{0}$ with $M_{1}=200 \mathrm{GeV}$ and $\tan \beta=10$. The $\Phi_{\mu}$ is set at $\pi$, i.e., negative $\mu$. Note that $\operatorname{BR}\left(\widetilde{\chi}_{3}^{0} \rightarrow \widetilde{\chi}_{2}^{0} \gamma\right)$ is very close to 0 . 
where $m_{i}$ denotes the mass of $\widetilde{\chi}_{i}^{0}$ or $\tilde{\chi}_{i}^{-}$and $X=Z, W$.

The helicity amplitude for neutralino-pair production is

$$
T\left(q \bar{q} \rightarrow \widetilde{\chi}_{i}^{0} \widetilde{\chi}_{j}^{0}\right)=\frac{e^{2}}{\hat{s}} \sum_{\alpha, \beta=L, R} \mathcal{Q}_{\alpha \beta}^{(\mathrm{nn}) i j}\left[\bar{v}(\bar{q}) \gamma_{\mu} P_{\alpha} u(q)\right]\left[\bar{u}\left(\widetilde{\chi}_{i}^{0}\right) \gamma^{\mu} P_{\beta} v\left(\widetilde{\chi}_{j}^{0}\right)\right]
$$

where $\alpha, \beta=L, R$ and (nn) in the superscript of $\mathcal{Q}_{\alpha \beta}^{(\mathrm{nn}) i j}$ denote neutralino pair production. The four generalized bilinear charges $\mathcal{Q}_{\alpha \beta}^{(\mathrm{nn}) i j}$ are

$$
\mathcal{Q}_{\alpha \beta}^{(\mathrm{nn}) i j}=\frac{D_{Z}}{s_{W}^{2} c_{W}^{2}} g_{\alpha}^{q} Q_{\beta i j}^{\mathrm{nnZ}} .
$$

Here the chiral couplings of the quark $q$ with the $Z$ boson are given by

$$
g_{R}^{q}=-s_{W}^{2} Q_{q}, \quad g_{L}^{q}=\left(T_{3}^{q}\right)_{L}-s_{W}^{2} Q_{q}
$$

where $\left(T_{3}^{q}\right)_{L}$ is the third component of the isospin and $Q_{q}$ is the electric charge of the quark $q$. The helicity amplitude for chargino pair production is

$$
T\left(q \bar{q} \rightarrow \widetilde{\chi}_{i}^{-} \widetilde{\chi}_{j}^{+}\right)=\frac{e^{2}}{\hat{s}} \sum_{\alpha, \beta} \mathcal{Q}_{\alpha \beta}^{(\mathrm{cc}) i j}\left[\bar{v}(\bar{q}) \gamma_{\mu} P_{\alpha} u(q)\right]\left[\bar{u}\left(\widetilde{\chi}_{i}^{-}\right) \gamma^{\mu} P_{\beta} v\left(\widetilde{\chi}_{j}^{+}\right)\right]
$$

where the bilinear charges are given by

$$
\mathcal{Q}_{\alpha \beta}^{(\mathrm{cc}) i j}=-Q^{q} \delta_{i j}+\frac{D_{Z}}{s_{W}^{2} c_{W}^{2}} g_{\alpha}^{q} Q_{\beta i j}^{\mathrm{ccZ}}
$$

Finally, the helicity amplitude for chargino-neutralino associated production is

$$
T\left(d \bar{u} \rightarrow \widetilde{\chi}_{i}^{-} \widetilde{\chi}_{j}^{0}\right)=\frac{e^{2}}{\hat{s}} \sum_{\alpha, \beta} \mathcal{Q}_{\alpha \beta}^{(\mathrm{cn}) i j}\left[\bar{v}(\bar{u}) \gamma_{\mu} P_{\alpha} u(d)\right]\left[\bar{u}\left(\widetilde{\chi}_{i}^{-}\right) \gamma^{\mu} P_{\beta} v\left(\widetilde{\chi}_{j}^{0}\right)\right]
$$

where the bilinear charges are

$$
\mathcal{Q}_{\alpha \beta}^{(\mathrm{cn}) i j}=\frac{D_{W}}{\sqrt{2} s_{W}^{2}} \delta_{\alpha L} Q_{\beta i j}^{\mathrm{cnW}}
$$

At parton level, the differential cross sections in the center-of-mass (c.m.) frame for the above three channels have a common expression

$$
\begin{aligned}
\frac{\mathrm{d} \sigma}{\mathrm{d} \cos \theta^{*}}= & \frac{1}{N_{c}} \frac{1}{\mathcal{S}} \frac{\pi \alpha^{2}}{2 \hat{s}} \lambda^{1 / 2} \\
& \times\left[\left\{1-\left(\mu_{i \hat{s}}-\mu_{j \hat{s}}\right)^{2}+\lambda \cos ^{2} \theta^{*}\right\} \mathcal{Q}_{1}^{i j}+4 \sqrt{\mu_{i \hat{s}} \mu_{j \hat{s}}} \mathcal{Q}_{2}^{i j}+2 \lambda^{1 / 2} \mathcal{Q}_{3}^{i j} \cos \theta^{*}\right]
\end{aligned}
$$


where $\theta^{*}$ is the scattering angle in the parton rest frame, $\lambda \equiv \lambda\left(1, \mu_{i \hat{s}}, \mu_{j \hat{s}}\right), N_{C}$ is the color factor of $q, \mathcal{S}$ is the symmetric factor $\left(\mathcal{S}=2\right.$ for $\widetilde{\chi}_{i}^{0} \widetilde{\chi}_{i}^{0}$ production) and $\theta^{*}$ is the scattering angle in the c.m. frame. The $\mathcal{Q}_{1,2,3}^{i j}$ are combinations of bilinear charges given by

$$
\begin{aligned}
\mathcal{Q}_{1}^{i j} & =\frac{1}{4}\left[\left|\mathcal{Q}_{R R}^{i j}\right|^{2}+\left|\mathcal{Q}_{L L}^{i j}\right|^{2}+\left|\mathcal{Q}_{R L}^{i j}\right|^{2}+\left|\mathcal{Q}_{L R}^{i j}\right|^{2}\right], \\
\mathcal{Q}_{2}^{i j} & =\frac{1}{2} \Re \mathrm{e}\left[\mathcal{Q}_{R R}^{i j} \mathcal{Q}_{R L}^{i j *}+\mathcal{Q}_{L L}^{i j} \mathcal{Q}_{L R}^{i j *}\right], \\
\mathcal{Q}_{3}^{i j} & =\frac{1}{4}\left[\left|\mathcal{Q}_{R R}^{i j}\right|^{2}+\left|\mathcal{Q}_{L L}^{i j}\right|^{2}-\left|\mathcal{Q}_{R L}^{i j}\right|^{2}-\left|\mathcal{Q}_{L R}^{i j}\right|^{2}\right],
\end{aligned}
$$

where the production channel of $(\mathrm{nn}),(\mathrm{cc})$ or $(\mathrm{cn})$ in the superscript is omitted.

In Fig. [7] we show the production cross sections for $\widetilde{\chi}_{3}^{0}$ at the LHC versus negative and positive $\mu$ with $\left|M_{1}\right|=200 \mathrm{GeV}$ and $\tan \beta=10$, including $\widetilde{\chi}_{3}^{0} \widetilde{\chi}_{1}^{0}, \widetilde{\chi}_{3}^{0} \widetilde{\chi}_{2}^{0}, \widetilde{\chi}_{3}^{0} \widetilde{\chi}_{1}^{+}$, and $\widetilde{\chi}_{3}^{0} \widetilde{\chi}_{1}^{-}$. Here we consider only the $C P$-conserving case $\left(\Phi_{\mu}=\pi, 0\right)$. It is clear that the cross section is in general larger in the region $|\mu| \sim M_{1}$, because this is region where the Bino and Higgsino mix strongly. This is also the favored parameter space for the Bino-Higgsino dark matter [5, 11, 12]. Away from the peak the cross sections in general decrease as the mixing between the Bino and the Higgsino becomes less; in particular, as $|\mu|$ decreases from the mixing region $\left(M_{1} \sim \mu\right)$ the $\widetilde{\chi}_{1}^{0}$ and $\widetilde{\chi}_{2}^{0}$ become Higgsino-like while $\widetilde{\chi}_{3}^{0}$ becomes Bino-like, and so the production of $\widetilde{\chi}_{3}^{0}$ with $\widetilde{\chi}_{1,2}^{0}$ and $\widetilde{\chi}_{1}^{ \pm}$all decrease rapidly.

The inclusive cross section for $\widetilde{\chi}_{3}^{0}$ is of the order of $O(0.1-0.2)$ pb for $M_{1}=200 \mathrm{GeV}$. We have also calculated that for $M_{1}=100 \mathrm{GeV}$ and maintaining the gaugino mass unification condition, the inclusive cross section for $\widetilde{\chi}_{3}^{0}$ is as large as $O(1-5) \mathrm{pb}$, while the cross section goes down to about $0.05 \mathrm{pb}$ for $M_{1}=300 \mathrm{GeV}$. Since we are interested in the radiative decay of $\widetilde{\chi}_{3}^{0}$, which could have a branching ratio as large as $O(1) \%$, the number of single photon plus $E_{T}$ events at the LHC is of the order of $O\left(10^{3}-5 \cdot 10^{3}\right), O(100-200)$, and $O(50)$ for $M_{1}=100,200,300 \mathrm{GeV}$, respectively. The next-to-leading order (NLO) SUSY corrections to gaugino pair production have been performed [28]. The $K$ factor is about $1.1-1.4$ depending on SUSY parameters. Since these SUSY corrections would not affect significantly the photon momentum or missing energy distributions, we just note that the event rates can be enhanced by up to about $40 \%$ due to NLO corrections.

Here we present the differential cross section versus the transverse momentum of the single-photon and versus the missing transverse momentum. We adopt a simple two-body decay of the third neutralino into the lightest neutralino and the photon, without taking into account the spin correlation, which should only be a mild effect. We also assume the decay is 

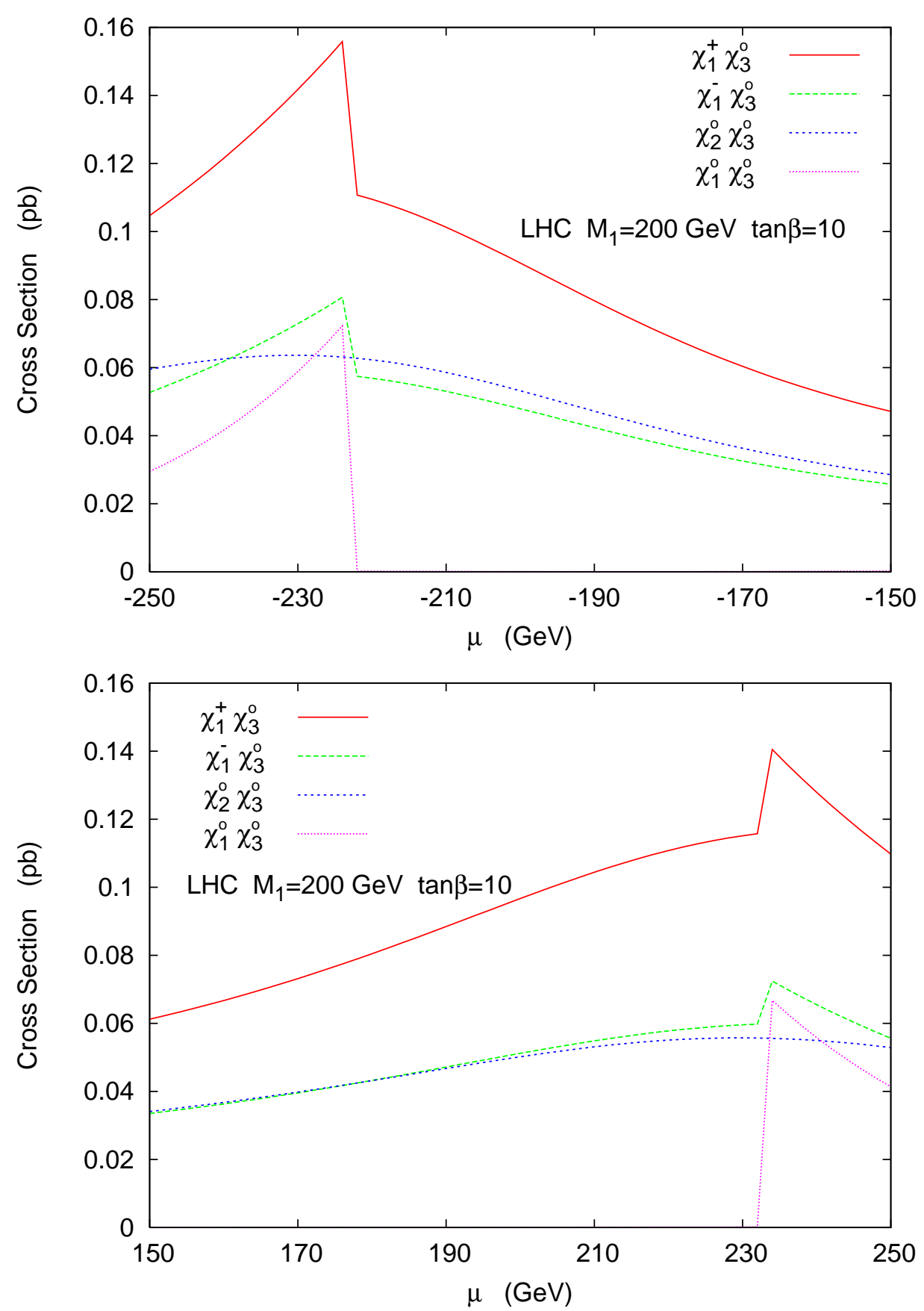

FIG. 7: Production cross sections for $\widetilde{\chi}_{3}^{0}$ at the LHC versus (a) negative and (b) positive $\mu$ with $M_{1}=200 \mathrm{GeV}$ and $\tan \beta=10$.

prompt, because the decay width of $\widetilde{\chi}_{3}^{0}$ is of the order of $\mathrm{MeV}$. The contributing production channels include $\widetilde{\chi}_{3}^{0} \widetilde{\chi}_{2}^{0}, \widetilde{\chi}_{3}^{0} \widetilde{\chi}_{1}^{0}$, and $\widetilde{\chi}_{3}^{0} \widetilde{\chi}_{1}^{ \pm}$, We include all of these production channels to account for inclusive $\widetilde{\chi}_{3}^{0}$ production. We focus on the radiative decay of $\widetilde{\chi}_{3}^{0} \rightarrow \widetilde{\chi}_{1}^{0} \gamma$, which has a branching ratio of the order of $1 \%$ in the region $|\mu| \sim M_{1}$. The charginos can decay 


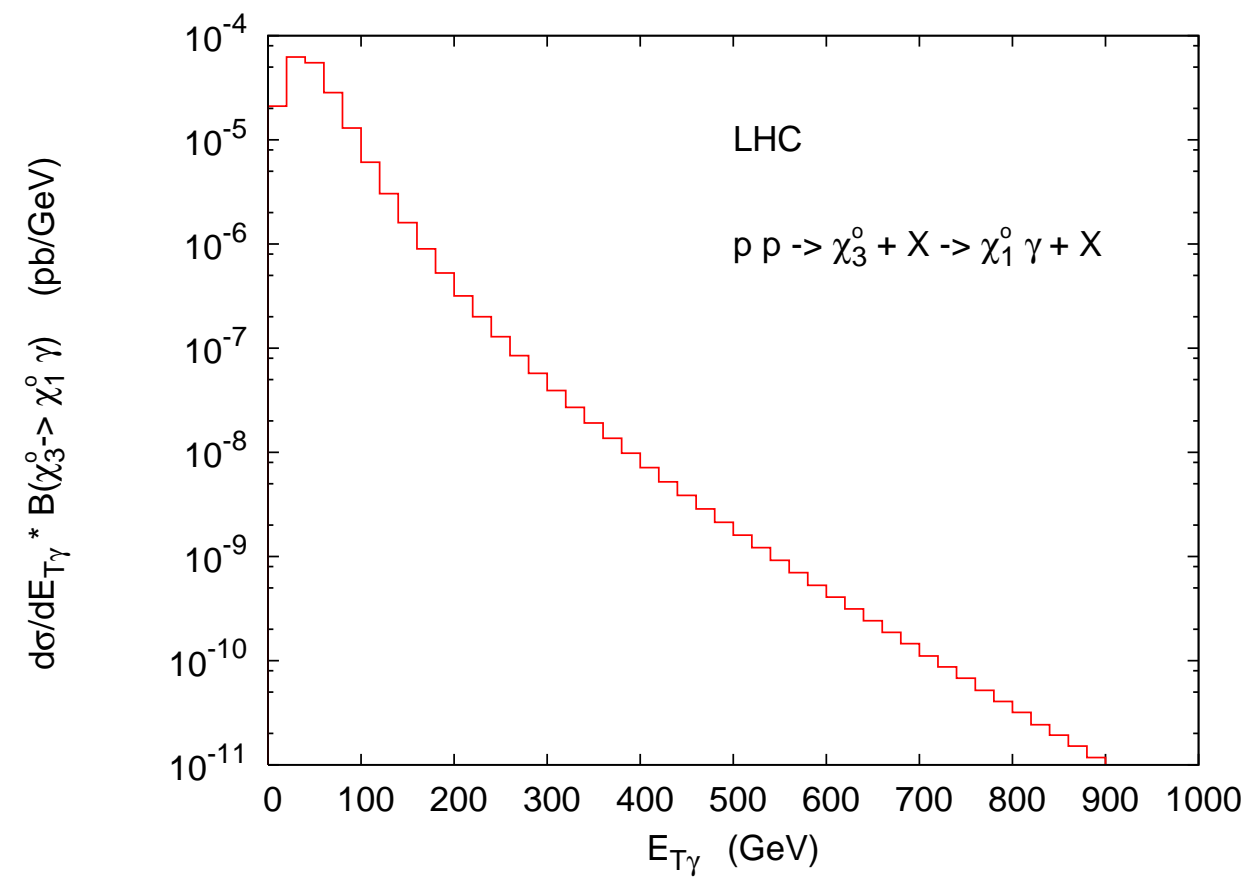

FIG. 8: Differential cross section versus the transverse momentum of the photon for the process $p p \rightarrow \widetilde{\chi}_{3}^{0}+X \rightarrow \widetilde{\chi}_{1}^{0} \gamma+X$. We set $M_{1}=200 \mathrm{GeV}, \mu=-220 \mathrm{GeV}$, and $\mathrm{BR}=1.68 \%$.

into the neutralino and a virtual $W$ boson, which further goes into $q \bar{q}^{\prime}$ or $\ell \nu_{\ell}$. Therefore, the final state consists of an isolated single-photon with or without charged leptons or jets, plus missing energies. We are primarily interested in the photon and missing energy distributions, which are shown in Fig. 8 and Fig. 9] respectively.

Here we give a brief discussion on possible backgrounds. An irreducible background comes from the SM production of $\gamma Z$ followed by $Z \rightarrow \nu \bar{\nu}$, as well as reducible background coming from quark fragmentation into a photon. Photon isolation and large $\not p_{T}$ cuts should be able to suppress the quark fragmentation background. The $\gamma Z$ background, on average, has a smaller missing energy than the signal, because very often in the signal there are two missing neutralino LSP's. Furthermore, a large portion of the signal will have a charged lepton or jets coming from the associated chargino decay that one can tag so as to remove the $\gamma Z$ background. Therefore, with the above mentioned cuts, tagging, and isolations, the signal of an isolated single-photon plus missing energy signature should be rather clean. 


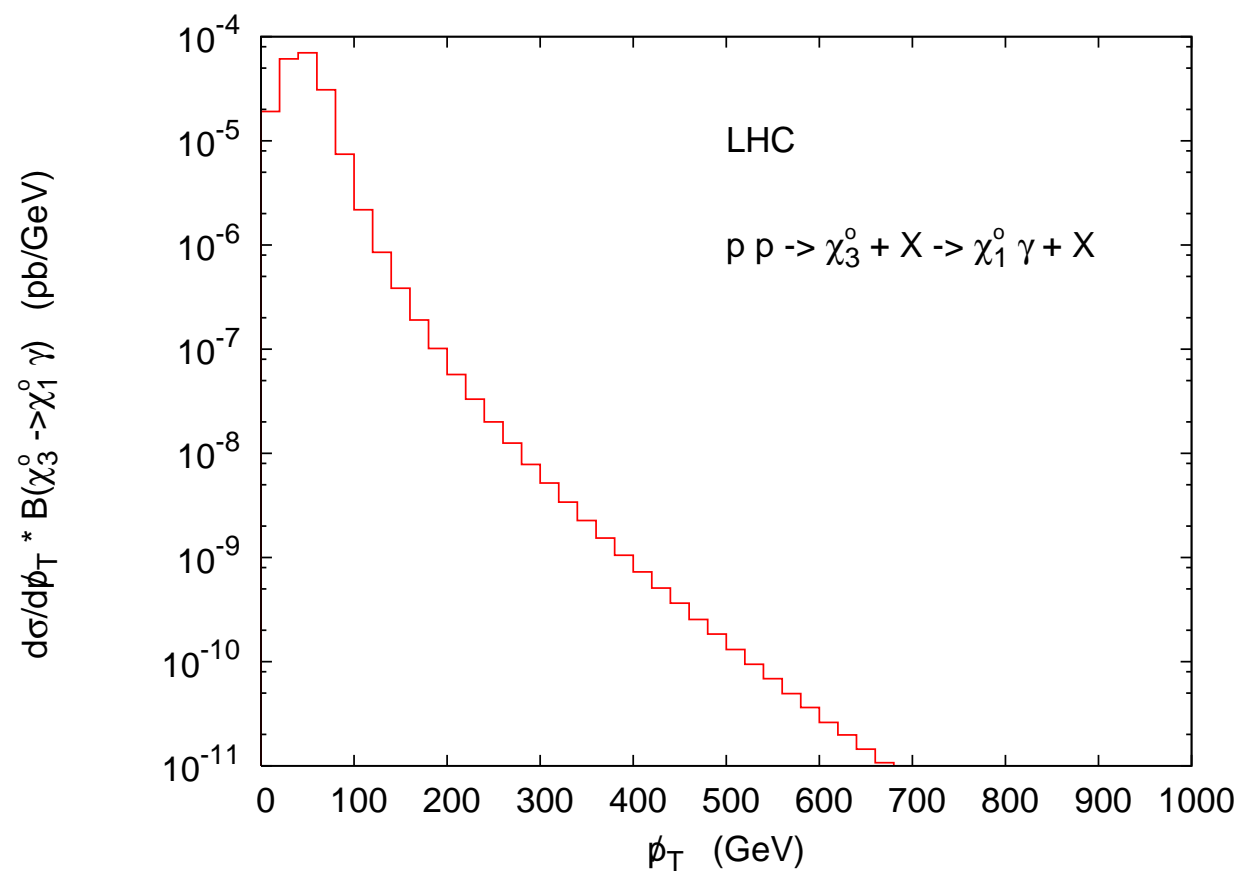

FIG. 9: Differential cross section versus the missing transverse momentum for the process $p p \rightarrow$ $\widetilde{\chi}_{3}^{0}+X \rightarrow \widetilde{\chi}_{1}^{0} \gamma+X$. We set $M_{1}=200 \mathrm{GeV}, \mu=-220 \mathrm{GeV}$, and $\mathrm{BR}=1.68 \%$.

\section{CONCLUSIONS}

We note that the single-photon plus missing energy signal is a possible sign of split SUSY, but not a unique feature. For example, gauge-mediated SUSY breaking (GMSB) models often predict various signals consisting of single- or multi-photon plus multiple leptons and jets, and missing energy. Split SUSY has to be established by gluino production, decay, and detection, and also with neutralino and chargino production and decay. One has to determine the sfermion mass scale and the $\mu$ parameter by simultaneously measuring the production and the decay properties of gauginos. One can use the mass spectrum and the photon energy spectrum to differentiate between split SUSY and GMSB models. We have shown that in general the $\widetilde{\chi}_{3}^{0} \rightarrow \widetilde{\chi}_{1}^{0} \gamma$ has a branching ratio of the order of $1 \%$, and the photon energy spectrum depends on the mass difference. On the other hand, in the GMSB models when the NLSP is a neutralino, the branching ratio into a photon and a gravitino is dominant. Therefore, the frequency of multi-photon plus missing energy events is very high. Also, the photon energy spectrum depends on the mass of the lightest neutralino only.

In summary, we have studied the decays and productions of neutralinos and charginos 
at hadron colliders in the scenario of split SUSY. The decays of neutralinos are particularly interesting because the sfermions are so heavy that only the decays via $Z^{(*)}, W^{(*)}$, and $h^{(*)}$ are possible, among which the radiative decay could have a branching ratio as large as $O(1) \%$, unlike the case of MSSM. We have found that $\widetilde{\chi}_{3}^{0} \rightarrow \widetilde{\chi}_{1}^{0} \gamma$ has a sufficiently large branching ratio when $|\mu| \sim M_{1}$ such that isolated single-photon plus missing energy events may be accessible in the LHC experiments. We argued that such events are rather clean and would be signs of split supersymmetry. Furthermore, counting the event rates will also give a measurement on the radiative decay branching ratio so that it gives information about the parameters of split SUSY.

\section{Acknowledgments}

We appreciate the hospitality of KIAS as we completed a part of this paper while we visit KIAS. The work of KC was supported in part by the National Science Council of Taiwan R.O.C. under grant no. NSC 93-2112-M-007-025-. The work of J. Song is supported by KRF under grant no. R04-2004-000-10164-0.

\section{APPENDIX A: COUPLINGS OF NEUTRALINOS AND CHARGINOS}

All the couplings of supersymmetric fermions, neutralinos and charginos, to gauge bosons

or Higgs bosons are expressed by $Q_{\alpha i j}^{f f^{\prime} X}$. Here $f\left(f^{\prime}\right)=\mathrm{c}$ in the superscript denotes the chargino, $f\left(f^{\prime}\right)=\mathrm{n}$ denotes the neutralino, $X=Z^{\mu}, W^{\mu}, h^{0}, H^{ \pm}, G^{ \pm}$, and $\alpha=L, R$ in the subscript is the chirality of the fermions. The $G^{ \pm}$is the Goldstone boson absorbed by $W^{ \pm}$ boson. In our notation, negatively charged chargino is assigned to be a particle such that its electric charge $Q_{\widetilde{\chi}^{ \pm}}$is -1 .

The specific expressions for the $\tilde{\chi}_{i}^{-}-\widetilde{\chi}_{j}^{+}-Z$ couplings are

$$
\begin{array}{rlrl}
Q_{L 11}^{\mathrm{ccZ}} & =s_{W}^{2}-\frac{3}{4}-\frac{1}{4} \cos 2 \phi_{L}, & Q_{R 11}^{\mathrm{ccZ}} & =s_{W}^{2}-\frac{3}{4}-\frac{1}{4} \cos 2 \phi_{R}, \\
Q_{L 12}^{\mathrm{ccZ}} & =-\frac{1}{4} \mathrm{e}^{-i \beta_{L}} \sin 2 \phi_{L}, & Q_{R 12}^{\mathrm{ccZ}}=-\frac{1}{4} \mathrm{e}^{-i\left(\beta_{R}-\gamma_{1}+\gamma_{2}\right)} \sin 2 \phi_{R}, \\
Q_{L 22}^{\mathrm{ccZ}}=s_{W}^{2}-\frac{3}{4}+\frac{1}{4} \cos 2 \phi_{L}, & Q_{R 22}^{\mathrm{ccZ}}=s_{W}^{2}-\frac{3}{4}+\frac{1}{4} \cos 2 \phi_{R} .
\end{array}
$$

The $\widetilde{\chi}_{i}^{0}-\widetilde{\chi}_{j}^{0}-Z$ couplings are

$$
Q_{L i j}^{\mathrm{nnZ}}=\frac{N_{i 3} N_{j 3}^{*}-N_{i 4} N_{j 4}^{*}}{2},
$$


where $Q_{R i j}^{\mathrm{nnZ}}=-\left(Q_{L i j}^{\mathrm{nnZ}}\right)^{*}$. Neutral couplings of $\widetilde{\chi}_{i}^{0}-\widetilde{\chi}_{j}^{0}-h^{0}$, where $h^{0}$ is the light $C P$-even Higgs boson, are divided into $i=j$ and $i \neq j$ cases, by using Majorana conditions:

$$
\begin{aligned}
S_{i}^{\mathrm{nnh}} & =\frac{1}{2}\left[N_{i 2}-t_{W} N_{i 1}\right]\left[-\sin \alpha N_{i 3}-\cos \alpha N_{i 4}\right], \\
Q_{L i j}^{\mathrm{nnh}} & =\frac{1}{2}\left[\left(N_{i 2}^{*}-t_{W} N_{i 1}^{*}\right)\left(-\sin \alpha N_{j 3}^{*}-\cos \alpha N_{j 4}^{*}\right)+(i \leftrightarrow j)\right],
\end{aligned}
$$

where $Q_{R i j}^{\mathrm{nnh}}=\left(Q_{L i j}^{\mathrm{nnh}}\right)^{*}, t_{W}=\tan \theta_{W}$ and

$$
\tan \alpha=\frac{m_{h}^{2}-m_{A}^{2} \cos ^{2} \beta-m_{Z}^{2} \sin ^{2} \beta}{\left(m_{A}^{2}+m_{Z}^{2}\right) \sin \beta \cos \beta} .
$$

The $\tilde{\chi}_{i}^{-}-\widetilde{\chi}_{j}^{0}-W^{+}$couplings are

$$
Q_{L i j}^{\mathrm{cnW}}=\left(U_{L}\right)_{i 1} N_{j 2}^{*}+\frac{1}{\sqrt{2}}\left(U_{L}\right)_{i 2} N_{j 3}^{*}, \quad Q_{R i j}^{\mathrm{cnW}}=\left(U_{R}\right)_{i 1} N_{j 2}-\frac{1}{\sqrt{2}}\left(U_{R}\right)_{i 2} N_{j 4} .
$$

The charged couplings to $H^{ \pm}$are

$$
\begin{aligned}
Q_{L k j}^{\mathrm{cnH}} & =\cos \beta\left[\left(U_{R}\right)_{k 1} N_{j 4}^{*}+\frac{\left(U_{R}\right)_{k 2}}{\sqrt{2}}\left(N_{j 2}^{*}+t_{W} N_{j 1}^{*}\right)\right], \\
Q_{R k j}^{\mathrm{cnH}} & =\sin \beta\left[\left(U_{L}\right)_{k 1} N_{j 3}-\frac{\left(U_{L}\right)_{k 2}}{\sqrt{2}}\left(N_{j 2}+t_{W} N_{j 1}\right)\right] .
\end{aligned}
$$

Finally we have the $\widetilde{\chi}_{i}^{-}-\widetilde{\chi}_{j}^{0}-G^{+}$couplings of

$$
Q_{\alpha k j}^{\mathrm{cnG}}=Q_{\alpha k j}^{\mathrm{cnh}}(\sin \beta \rightarrow-\cos \beta, \quad \cos \beta \rightarrow \sin \beta)
$$

[1] N. Arkani-Hamed and S. Dimopoulos, hep-th/0405159.

[2] S. Weinberg, "Anthropic Bound On The Cosmological Constant," Phys. Rev. Lett. 59, 2607 (1987); A. Vilenkin, "Predictions from quantum cosmology," Phys. Rev. Lett. 74, 846 (1995).

[3] R. Bousso and J. Polchinski, "Quantization of four-form fluxes and dynamical neutralization of the cosmological constant," JHEP 0006, 006 (2000); J.L. Feng, J. March-Russell, S. Sethi, and F. Wilczek Nucl. Phys. B602, 307 (2001); S. Kachru, R. Kallosh, A. Linde and S. P. Trivedi, "De Sitter vacua in string theory," Phys. Rev. D 68, 046005 (2003); L. Susskind, "The anthropic landscape of string theory," arXiv:hep-th/0302219, F. Denef and M. R. Douglas, "Distributions of flux vacua," JHEP 0405, 072 (2004).

[4] C. L. Bennett et al., Astrophys. J. Suppl. 148, 1 (2003); D. N. Spergel et al., Astrophys. J. Suppl. 148, 175 (2003). 
[5] G. Giudice and A. Romanino, Nucl. Phys. B 699, 65 (2004). [Erratum-ibid. B 706, 65 (2005)] arXiv:hep-ph/0406088.

[6] N. Arkani-Hamed, S. Dimopoulos, G. F. Giudice and A. Romanino, Nucl. Phys. B 709, 3 (2005) arXiv:hep-ph/0409232.

[7] D. Chang, W. F. Chang and W. Y. Keung, D. Chang, W. F. Chang and W. Y. Keung, Phys. Rev. D 71, 076006 (2005); N. G. Deshpande and J. Jiang, Phys. Lett. B 615, 111 (2005).

[8] A. Arvanitaki, C. Davis, P. W. Graham and J. G. Wacker, Phys. Rev. D 70, 117703 (2004) arXiv:hep-ph/0406034;

[9] M. Binger, arXiv:hep-ph/0408240

[10] R. Mahbubani, arXiv:hep-ph/0408096.

[11] A. Pierce, Phys. Rev. D 70, 075006 (2004).

[12] A. Masiero, S. Profumo and P. Ullio, Nucl. Phys. B 712, 86 (2005).

[13] OPAL Coll. (G. Abbiendi et al.), Eur. Phys. J. C35, 1 (2004).

[14] W. Kilian, T. Plehn, P. Richardson and E. Schmidt, Eur. Phys. J. C 39, 229 (2005).

[15] J. L. Hewett, B. Lillie, M. Masip and T. G. Rizzo, JHEP 0409, 070 (2004) arXiv:hep-ph/0408248;

[16] L. Anchordoqui, H. Goldberg and C. Nunez, Phys. Rev. D 71, 065014 (2005).

[17] K. Cheung and W. Y. Keung, Phys. Rev. D 71, 015015 (2005).

[18] S. h. Zhu, Phys. Lett. B 604, 207 (2004).

[19] K. Cheung and C.W. Chiang, Phys. Rev. D 71, 095003 (2005).

[20] N. Arkani-Hamed, S. Dimopoulos, and S. Kachru, arXiv:hep-th/0501082.

[21] P. Fox et al., arXiv:hep-th/0503249.

[22] J. L. Feng and F. Wilczek, arXiv:hep-ph/0507032.

[23] J. D. Wells, Phys. Rev. D 71, 015013 (2005) arXiv:hep-ph/0411041.

[24] S. Y. Choi, J. Kalinowski, G. Moortgat-Pick and P. M. Zerwas, Eur. Phys. J. C 22, 563 (2001) [Addendum-ibid. C 23, 769 (2002)].

[25] S. Y. Choi, A. Djouadi, M. Guchait, J. Kalinowski, H. S. Song and P. M. Zerwas, Eur. Phys. J. C 14, 535 (2000).

[26] H. E. Haber and G. L. Kane, Phys. Rept. 117, 75 (1985).

[27] H. Haber and D. Wyler, Nucl. Phys. B323, 267 (1989).

[28] W. Beenakker, R. Hopker, and M. Spira, hep-ph/9611232; W. Beenakker, M. Klasen, M. 
Kramer, T. Plehn, M. Spira, and P. Zerwas, Phys. Rev. Lett. 83, 3780 (1999). 